\title{
Jurnal
}

Ilmu Manajemen dan Bisnis

Terakreditasi Kemenristekdikti Nomor 34/E/KPT/ Desember 2018

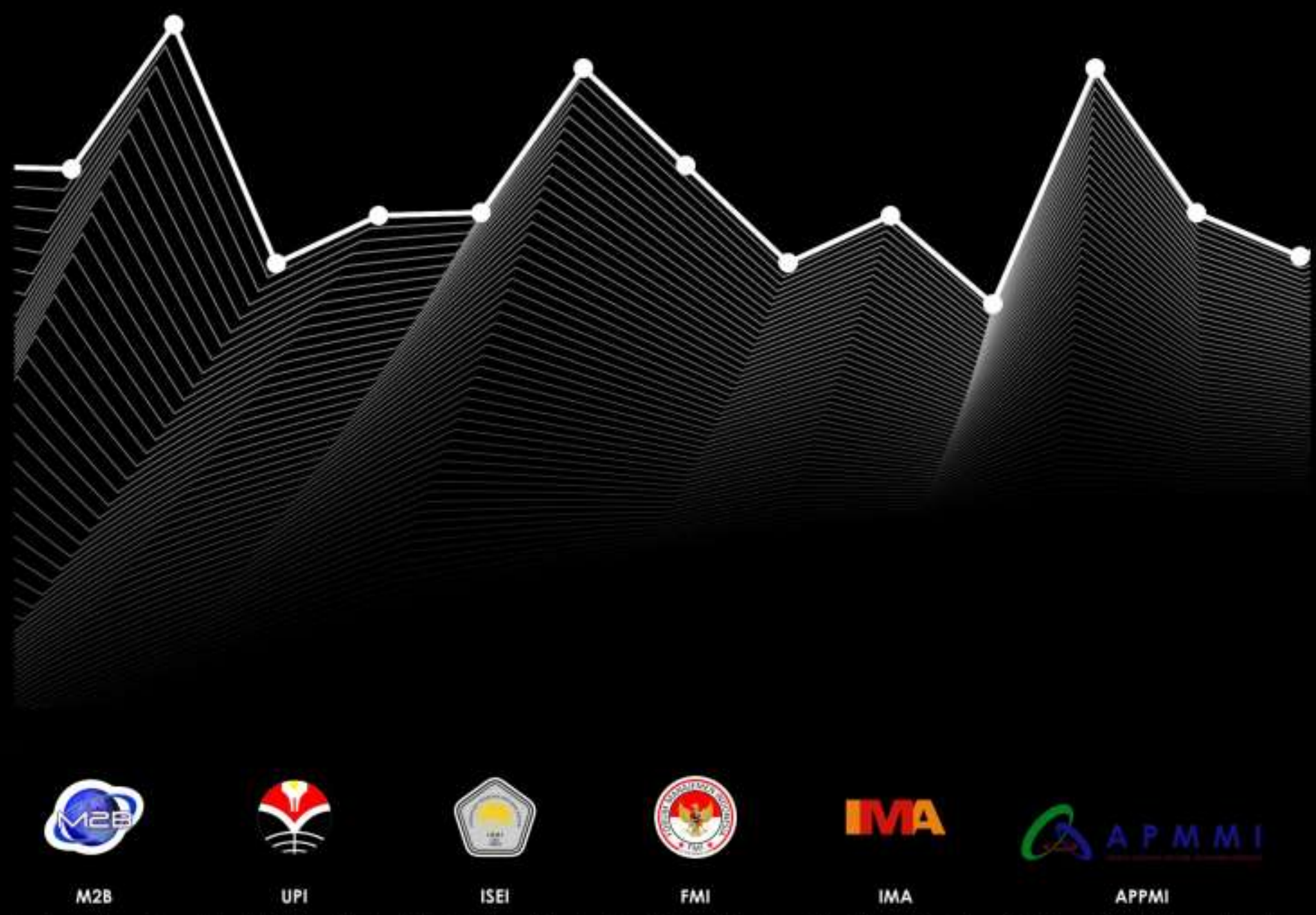


The aim of this Jurnal Ilmu Manajemen dan Bisnis is to promote a principled approach to research on management science and business related concerns by encouraging inquiry into the relationship between theoretical and practical studies. Jurnal Ilmu Manajemen dan Bisnis, an electronic journal, provides a forum for publishing the original research articles, review articles from contributors, and the novel technology news related to management science and business.

Further, the journal is intended as an instrument for individuals conducting research on management science and business at both micro and macro levels of analysis as well as its relationship with other managerial and business functions.

Editorial Team welcome submissions of papers describing researchers, practitioners, regulators, students, and other parties interested in the development of management science and business. Accepts manuscripts of either quantitative research, qualitative research, mix method research, and Research and Development (R\&D) written in either Bahasa Indonesia or English.

The scopes of the topics include OrganzationL Behavior, Leadership, Human Resources Management, Innovation, IT, Operations and Supply Chain Management, Marketing Management, Financial Management, Accounting, Strategic Management, Entrepreneurship, and Green Business.

\section{PUBLICATION INFORMATION}

Jurnal Ilmu Manajemen dan Bisnis with registered number ISSN 2337-411X (Print) and ISSN 2503-3522 (Online), is a peer-reviewed journal published two times a year (March and September) by Management Program Sekolah Pascasarjana Universitas Pendidikan Indonesia. Jurnal Ilmu Manajemen dan Bisnis is intended to be the journal for publishing articles reporting the results of research on business. Jurnal Ilmu Manajemen dan Bisnis cooperation with Ikatan Sarjana Ekonomi Indonesia (ISEI) Jawa Barat, Forum Manajemen Indonesia (FMI), Indonesia Marketing Associations, Asosiasi Pengelola Magister Manajemen Indonesia (APMMI). DOI Prefix 10.17509/jimb by Crossref

\section{OPEN ACCES POLICY}

The article submitted to this online journal will be peer-reviewed at least 2 (two) reviewers. This journal provides immediate open access to its content on the principle that making research freely available to the public supports a greater global exchange of knowledge. Before going to review process, all manuscripts will be checked that they are free from plagiarism practice using "Turnitin" software. If there an indication of plagiarism, the manuscript will instantly be rejected. Indeks by Google Scholar and Indonesia One Search. Nationally Accredited based on the Decree of the Minister of Research, Technology and Higher Education, Number 34/E/KPT/2018 - 10 December 2018 


\section{EDITORIAL BOARD}

\section{Editor in Chief}

Prof. Dr. Hj. Ratih Hurriyati, MP, ID SCOPUS : 57074106300, Fakultas Pendidikan Ekonomi dan Bisnis, Universitas Pendidikan Indonesia, Indonesia, Indonesia

\section{Vice Editor}

Prof. Dr. H. Agus Rahayu, MP, ID SCOPUS : 57188870250, Fakultas Pendidikan Ekonomi dan Bisnis, Universitas Pendidikan Indonesia, Indonesia, Indonesia, Indonesia

Prof. Dr. H. Disman MS, ID SCOPUS : 57188870250, Fakultas Pendidikan Ekonomi dan Bisnis, Universitas Pendidikan Indonesia, Indonesia, Indonesia

\section{Editor}

Dr. Vanessa Gaffar, SE., Akt., MBA, ID SCOPUS : 57193739131, Fakultas Pendidikan Ekonomi dan Bisnis, Universitas Pendidikan Indonesia, Indonesia, Indonesia

Dr. Lili Adi Wibowo, S.Pd., S.Sos., MM, ID SCOPUS : 57193737268, Fakultas Pendidikan Ekonomi dan Bisnis, Universitas Pendidikan Indonesia, Indonesia, Indonesia, Indonesia

Dr. Heny Hendrayati, MM, ID SCOPUS : 57193444685, Fakultas Pendidikan Ekonomi dan Bisnis, Universitas Pendidikan Indonesia, Indonesia

Dr. Yana Setiawan, S.Pd., MM, Fakultas Pendidikan Ekonomi dan Bisnis, Universitas Pendidikan Indonesia, Indonesia, Indonesia

\section{Circulation and Distribution}

Usep Muharam, S.Pd, Program Studi Manajemen, Sekolah Pascasarjana, Universitas Pendidikan Indonesia 


\section{PEER REVIEWER}

Prof. Ikuro Yamamoto, Kinzo University, Japan

Prof. Je Dae SIK, Youngsan University, South Korea

Prof. Kim, Tae Hee, Youngsan University, South Korea

Prof. Lincoln Arsyad, M.Sc., Ph.D, SCOPUS ID: 54683353100, Fakultas Ekonomi dan Bisnis, Universitas Gajah Mada, Indonesia

Prof. Dr. Sukrisno Agoes., Ak., MM., CPA, ID SCOPUS :54683353100, Fakultas Ekonomi, Universitas Tarumanagara, Indonesia

Ina Primiana, ID SCOPUS: 55437876800 Fakultas Ekonomi dan Bisnis, Universitas Padjadjaran, Indonesia

Dr. Ir. H. Arry Akhmad Arman, MT, ID SCOPUS : 56039352800 Sekolah Teknik Elektro dan Informatika, Institut Teknologi Bandung, Indonesia

Dwi Larso, Ph.D M.Sc, ID SCOPUS : 34969340900, School of Business and Management,Institut Teknologi Bandung, Indonesia

Ari Waroka, Ph.D., M.Sc, MDEM., MCEUE, DEA, ID SCOPUS : 55385462400, Universidad Autonoma de Madrid, Madrid, Spain 


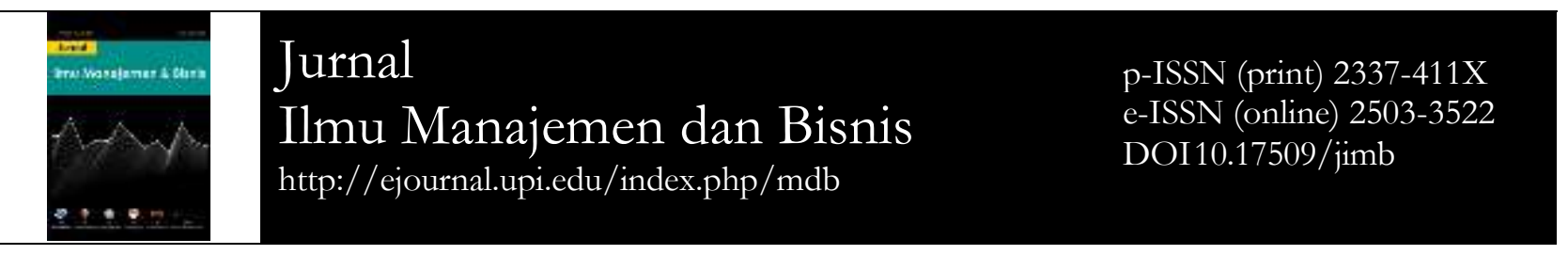

\section{DAFTAR ISI}

VOL 10 NO 1 MARET 2019

Organizational Culture and Intrapreneurship Employee of The Impact $1-8$ on Work Discipline of Employees in Brangkal Offset

Mirhamida Rahmah

Hospitality and Tourism Industry Performance in Indonesia Based on 9-16 Benjamin Graham's Perspective

Ima Kristina Yulita ${ }^{1}$ and Caecilia Wahyu Estining Rahayu ${ }^{2}$

Pengaruh Perilaku Kepemimpinan, Motivasi Berprestasi dan Budaya 17-28 Sekolah Terhadap Kinerja Guru

Tini Martini

Pengaruh Integrasi Rantai Pasokan Terhadap Keunggulan Bersaing

Melalui Kinerja Rantai Pasokan pada Peternak Sapi Perah di Kabupaten Bandung Barat

Yun Yun ${ }^{1}$. Asep Kurniawan²

Pengaruh Pemberian CSR Dana Pendidikan dari PT. Internusa Jaya 43-50 Sejahtera Terhadap Peningkatan Kesejahteraan Masyarakat Samrotu Sa'adah ${ }^{1}$, Elvira Azis ${ }^{2}$

Pengaruh Transparansi dan Akuntabilitas Terhadap Kinerja Instansi $\quad$ 51-62 Pemerintah

Ait Novatiani ${ }^{1}$, R. Wedi Rusmawan Kusumah², Diandra Pepi Vabiani3

Analisis Penggunaan E-Commerce Terhadap Peningkatan Permintaan Kuantitas Produk Pada Umkm Di Kabupaten Banyumas (Suatu Pendekatan Teori Technology Acceptance Model)

Dona Primasari ${ }^{1}$ Sudjono $^{2}$ Nining Abriani ${ }^{3}$

Pengaruh Penggunaan Metode Pembelajaran Group Investigation Terhadap Literasi Keuangan Dengan Moderator Kecerdasan $75-82$ Emosional

Azizah Fauziyah ${ }^{1}$, Disman $^{2}$, Kurjono $^{3}$

Penentuan Harga Opsi Barrier Menggunakan Metode Trinomial 83-92 Kamrad-Ritchken Dengan Volatilitas Model Garch

S.Sulastri ${ }^{1}$, Lienda Novieyanti ${ }^{2}$, Sukono ${ }^{3}$

Implementation of Bussniess Model Canvas in Designing Management 93-100 of JASKOST Application

Wanda Fatricia ${ }^{1}$, Mokh. Adib Sultan ${ }^{2}$ 


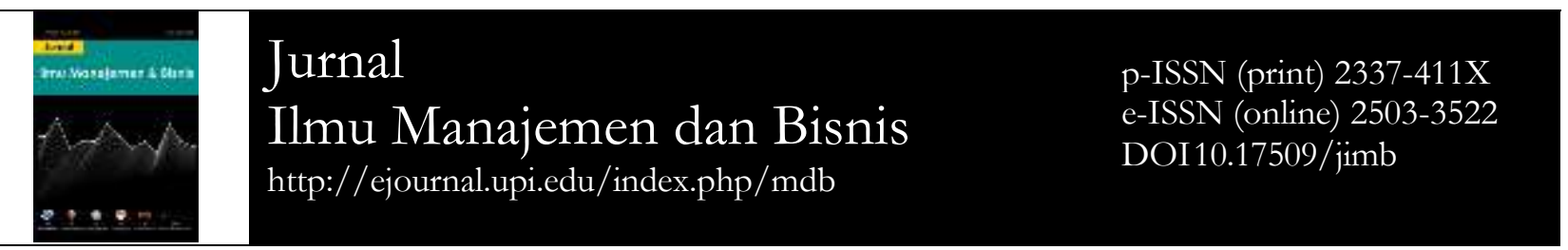

Marital Status dan Gender: Investigasi Kepuasan Kerja Karyawan 101-106 Hotel

Rian Andriani ${ }^{1}$, Disman

Analisis Manajemen Risiko Teknologi Informasi Pada Kasus Skimming $\quad$ 107-120 ATM Bank X

Wahyu Firmandani ${ }^{1}$, M. Malik ${ }^{2}$ 